\title{
L'« hiver du mécontentement » de 1978-1979: du mythe politique à la crise interne du mouvement travailliste
}

The "Winter of Discontent " of 1978-79: Political Myth and Internal Crisis of the Labour Movement

Marc Lenormand

\section{OpenEdition \\ Journals}

Édition électronique

URL : http://journals.openedition.org/rfcb/1683

DOI : $10.4000 /$ rfcb.1683

ISSN : 2429-4373

Éditeur

CRECIB - Centre de recherche et d'études en civilisation britannique

\section{Référence électronique}

Marc Lenormand, «L'« hiver du mécontentement » de 1978-1979 : du mythe politique à la crise interne du mouvement travailliste », Revue Française de Civilisation Britannique [En ligne], XXII- Hors série I 2017, mis en ligne le 30 décembre 2017, consulté le 10 décembre 2020. URL : http:// journals.openedition.org/rfcb/1683; DOI : https://doi.org/10.4000/rfcb.1683

Ce document a été généré automatiquement le 10 décembre 2020.

\section{(c) $(1) \odot$}

Revue française de civilisation britannique est mis à disposition selon les termes de la licence Creative Commons Attribution - Pas d'Utilisation Commerciale - Pas de Modification 4.0 International. 


\title{
L'« hiver du mécontentement » de 1978-1979: du mythe politique à la crise interne du mouvement travailliste
}

\author{
The "Winter of Discontent " of 1978-79: Political Myth and Internal Crisis of the \\ Labour Movement
}

Marc Lenormand

1 Quand on évoque l'« hiver du mécontentement » de 1978-1979, on parle en réalité de deux choses distinctes. Tout d'abord, l'« hiver du mécontentement » est un terme permettant d'englober un ensemble de conflits sociaux qui se déroulent de septembre 1978 à avril 1979, au cours desquels une série de groupes de travailleurs et de travailleuses et leurs syndicats contestent la politique de contrôle des revenus ${ }^{1}$ mise en place par le gouvernement travailliste depuis 1975, avec le soutien décroissant de la direction du Trades Union Congress (TUC), la confédération des syndicats britanniques. Les principaux épisodes en sont trois grèves nationales de grande ampleur dans les usines Ford de septembre à novembre 1978, dans les transports routiers en janvier et février 1979, enfin dans les services publics de janvier à avril 1979². Cependant, l'«hiver du mécontentement» est aussi un signifiant qui, par rapport aux conflits sociaux précédemment évoqués, a acquis une autonomie relative dès son émergence au cours de l'hiver 1978-1979, pour se voir attribuer toute une série de signifiés : le désordre social, la faiblesse du gouvernement, les excès du militantisme syndical, la faillite du consensus politique d'après-Guerre. Comme Tara Martin l'a souligné dans le chapitre qu'elle consacre aux luttes de mémoire autour des conflits de l'hiver 1978-1979, il est tout à fait remarquable qu'une telle vision hypostasiée de l' "hiver du mécontentement", immédiatement popularisée par le parti conservateur et ses relais dans la presse, se soit par la suite étendue non seulement à la fraction dite "modernisatrice» du parti travailliste souhaitant procéder à un recentrage du parti ${ }^{3}$, mais aussi à certains syndicalistes qui ont participé directement à ces conflits ${ }^{4}$. L'étude qui a fait date sur ce 
sujet est l'article que Colin Hay a consacré, en 1996, à ce qu'il nomme «la construction discursive de la crise $»^{5}$, c'est-à-dire le processus par lequel les évènements relativement disparates de l'hiver 1978-1979 ont été rassemblés pour constituer un ensemble cohérent, lequel a ensuite pu être interprété comme signe d'une « crise » de la gestion travailliste de l'économie britannique et faciliter l'accession des conservateurs à une hégémonie idéologique ${ }^{6}$, prélude à leur victoire politique aux élections à la Chambre des communes de mai 1979.

2 Il en résulte, au sujet de l'.« hiver du mécontentement ", une disjonction forte entre d'un côté la réalité complexe et la grande hétérogénéité des conflits sociaux, et de l'autre leur codification en un slogan aussi réducteur qu'évocateur. L'enjeu de cet article est donc de restituer une partie de la complexité de l'« hiver du mécontentement ", de donner sens aux conflits sociaux de l'hiver 1978-1979 en les inscrivant dans une série de dynamiques susceptibles d'apporter une compréhension de ce qui se passe au cours de l'«hiver du mécontentement» et de la portée de cet épisode: la transformation du mouvement syndical à partir de la fin des années 1960 ; l'évolution de la relation entre les ailes syndicales et parlementaire du mouvement travailliste entre 1970 et 1979 ; enfin, les tensions politiques et programmatiques très fortes qui traversent le parti travailliste pendant cette même période, et ne connaissent une résolution que dans les années 19807.

\section{L'apogée d'une décennie militante?}

3 Pour aborder les transformations du mouvement syndical au cours de la décennie, il semble utile d'en élargir les contours chronologiques pour inclure la toute fin des années 1960. La période 1966-1970 est en effet marquée par trois conflits très différents mais fortement médiatisés. La grève nationale des marins de la National Union of Seamen, au printemps 1966, constitue un premier défi lancé à la politique de contrôle des revenus du gouvernement Wilson, lequel réagit fermement en déclarant l'état d'urgence pour briser la grève, Wilson dénonçant une grève organisée par des agitateurs communistes ${ }^{8}$. Deux ans plus tard, les couturières de l'usine Ford de Dagenham, entraînant à leur suite celles de Halewood, se mettent en grève contre leur reclassement à un niveau de qualification et de rémunération inférieur à leurs collègues masculins, poussant le gouvernement à intercéder en leur faveur et à légiférer sous la forme de l'Equal Pay Act de 1970.

4 Au printemps 1969 enfin, des grèves spontanées éclatent après la publication du livre blanc In Place of Strife, qui vise à réguler par la loi l'activité syndicale. On voit émerger ici trois dynamiques qui se renforcent dans les années 1970, et constituent une rupture par rapport à la situation sociale de l'après-guerre : la mobilisation et le recours à la grève de catégories de travailleurs et de travailleuses occupant des positions subordonnées dans le monde du travail et jusqu'alors aux marges du mouvement syndical ; le retour des conflits nationaux opposant directement des syndicats à des gouvernements, alors que c'était la conflictualité locale qui prédominait depuis l'échec de la grève générale de 1926 ; enfin le déploiement d'un discours gouvernemental hostile à l'activité syndicale militante et de mesures visant à briser les grèves, là où les gouvernements travaillistes comme conservateurs de l'après-guerre avaient jusqu'alors adopté une attitude entre indifférence et conciliation à l'égard des syndicats. 


\section{Les caractéristiques de la conflictualité sociale dans les années 1970}

5 Ces dynamiques sont confirmées au cours de la décennie suivante, 1970-1979. Celle-ci s'ouvre sur une série de confrontations entre le gouvernement conservateur d'Edward Heath et des syndicats qui résistent à ses orientations : opposition à la criminalisation de l'activité syndicale introduite par l'Industrial Relations Act de 1971, qui aboutit à l'épisode des Pentonville Five, ces cinq dockers emprisonnés pour avoir bravé les décisions de la toute nouvelle National Industrial Relations Court, avant d'être finalement relâchés; résistance à l'imposition d'une logique de marché dans l'industrie, lorsque les travailleurs des chantiers navals de UCS (Upper Clyde Shipbuilders) sur la Clyde organisent un work-in pour maintenir l'activité sur leur site, et font finalement plier le gouvernement; lutte contre la mise en place d'un contrôle strict des revenus enfin, marquée par les deux grèves nationales organisées par la NUM (National Union of Mineworkers), le syndicat des mineurs, en 1972 et à l'hiver 1973-1974'. Le gouvernement conservateur semble bien être aux prises directement avec les organisations syndicales, et ces dernières l'emporter dans la plupart des confrontations. Si le milieu de la décennie est marqué par un reflux de la contestation sociale après l'arrivée au pouvoir des travaillistes en 1974, les conflits nationaux opèrent un retour à partir de l'automne 1977, face à une politique de contrôle des revenus qui réduit depuis deux ans le pouvoir d'achat des salariés : les contrôleurs aériens se mettent en grève, ainsi que les pompiers, dont le syndicat FBU (Fire Brigades Union) lance la première grève de son histoire ${ }^{10}$. Les conflits de l'hiver 1978-1979, portant sur la limitation des augmentations salariales à $5 \%$ dans un contexte de forte inflation, s'inscrivent dans la continuité de ces épisodes précédents.

Ces conflits suscitent la mobilisation d'un ensemble de dispositifs anti-syndicaux. Le recours à la loi, ébauché par le gouvernement Wilson et mis en œuvre par le gouvernement Heath, trouve certes ses limites lorsque l'Industrial Relations Act est neutralisé par l'action syndicale, mais le gouvernement dispose toujours d'un appareil d'État qui se renforce au cours de la décennie. Après que la police a échoué à empêcher le blocage de l'économie par les mineurs lors du conflit de l'hiver 1972, le gouvernement Heath met en place une Civil Contingencies Unit visant à briser les grèves : celle-ci est activée à deux reprises lorsque le gouvernement Callaghan déclare l'État d'urgence, fin 1977 contre les pompiers et de nouveau en janvier 1979 contre les chauffeurs de camionsciternes en Irlande du Nord. Cette mobilisation de l'appareil d'État contre les syndicats est légitimée et facilitée par le développement de discours anti-syndicaux. La dénonciation par les gouvernements de l'égoïsme des syndicats à un moment où l'ensemble du pays se serre la ceinture trouve un écho et des munitions dans la couverture des conflits sociaux par une presse très majoritairement conservatrice, qui stigmatise l'avidité et les abus de pouvoir des organisations syndicales. Cette rhétorique n'est certes pas toujours efficace, comme lorsque Heath dévoile la faiblesse de la position du gouvernement en demandant aux électeurs et aux électrices de trancher le conflit qui oppose le gouvernement à la NUM en février 1974. Toutefois, la virulence du programme anti-syndical que les conservateurs peuvent présenter au cours de l'hiver 1978-1979 est nourrie par une décennie de construction progressive d'un consensus anti-syndical au sein des élites britanniques, et sa traduction et diffusion par les médias auprès de la population. 
7 Enfin, la décennie est caractérisée par l'extension de la conflictualité sociale à de nouveaux secteurs et de nouveaux groupes de travailleurs et travailleurs. Si l'épisode le plus marquant à cet égard est sans nul doute la grève de Grunwick qui dure de 1976 à $1978^{11}$, les femmes et les immigrés sont également nombreux à occuper des positions subordonnées dans les services publics, qui ont connu une forte expansion dans l'aprèsguerre. Ces secteurs, traditionnellement peu prompts à recourir à la grève, sont radicalisés par la baisse de la rémunération pendant les périodes successives de contrôle des revenus, par la restructuration brutale des services mise en œuvre au début de la décennie et par les fermetures d'écoles et d'hôpitaux qui se multiplient dans le contexte de réduction budgétaire de la seconde moitié de la décennie ${ }^{12}$. Il en résulte une série de conflits au cours desquels ces groupes de travailleurs passent à l'action souvent pour la première fois: les conflits de l'« hiver du mécontentement» de 1978-1979 s'inscrivent ainsi dans la continuité de la "dirty jobs strike » lancée par les éboueurs en 1969, la grève dans les collectivités locales de 1970, la grève dans le NHS de 1972 et les nombreuses occupations et work-in qui marquent les luttes contre les fermetures d'hôpitaux à partir de $1976^{13}$.

\section{La diversité des conflits sociaux au cours de l'« hiver du mécontentement »}

8 Si ces dynamiques convergent au cours de l'hiver 1978-1979 pour donner ses caractéristiques à l'« hiver du mécontentement", on soulignera que chacun des trois principaux conflits de l'hiver manifestent des traits spécifiques à la situation du mouvement syndical dans les différents secteurs de l'économie britannique. Le conflit qui ouvre l'« hiver du mécontentement ", dans les usines Ford, présente des caractéristiques relativement traditionnelles, puisqu'après l'échec d'une première séance de négociations salariales entre la direction de Ford et les syndicats présents dans l'entreprise, ce sont les 18000 travailleurs de l'usine de Halewood qui prennent collectivement l'initiative de la grève en septembre 1978, entraînant à leur suite les directions syndicales et le reste des usines du groupe Ford au Royaume-Uni. Ce conflit illustre ainsi la force de l'organisation syndicale locale dans les ateliers et dans les usines, et plus largement la confiance des syndicats du secteur manufacturier dans leur capacité à faire plier des employeurs dont les profits dépendent de la reprise de la production.

9 Le deuxième conflit majeur de l'hiver, dans les transports routiers, conduit au même résultat sans pour autant prendre les mêmes formes. En dépit du caractère souvent solitaire du métier de routier, les syndicats se sont fortement développés dans le secteur au cours de la décennie et ont permis la constitution de collectifs militants forts dans les entreprises. Alors même que les employeurs, pressés en cela par le gouvernement, ne souhaitent faire que des concessions salariales limitées, les routiers s'organisent pour bloquer les infrastructures du pays et la circulation des marchandises - un blocage dont l'effet est redoutable lorsqu'il se combine comme c'est le cas en janvier 1979, avec des grèves dans les chemins de fer et un hiver particulièrement rude. Les routiers obtiennent la satisfaction de leurs revendications avant la fin du mois ${ }^{14}$. Par rapport à ces groupes de travailleurs confiant dans leur force collective et bénéficiant de moyens de blocage de la production ou plus largement de l'économie, les travailleurs et les travailleuses des services publics qui se mettent en grève le 22 janvier 1979 le font dans des conditions différentes, et selon des orientations définies par les directions des syndicats du secteur, 
soucieux de préserver leur image et de modérer l'impact de la grève autant sur les salariés que sur la population qui bénéficie des services publics. Ainsi, c'est davantage à une guérilla sociale que les syndicats du secteur se livrent entre janvier et mars 1979, espérant maintenir la pression sur le gouvernement par des grèves perlés qui affectent successivement les services des collectivités locales et du NHS. Ce dernier mouvement s'achève sur des concessions faibles de la part du gouvernement, sur des récriminations entre organisations syndicales et sur de fortes tensions entre une base militante radicalisée et des directions syndicales désireuses de trouver une issue qui soit favorable, à défaut d'être victorieuse ${ }^{15}$.

Il y a cependant un risque, en arrêtant cette étude en 1979, de voir l'«hiver du mécontentement » exclusivement comme l'apogée d'une décennie militante, prélude à une remise en ordre thatchérienne. Certes, après le nombre record de journées de grève enregistré en 1979, la conflictualité sociale baisse tout au long des années 1980 et 1990. Cependant, les facteurs d'une telle baisse du nombre de journées de grève sont multiples : 1979 marque aussi le début d'une période de récession - certes encouragée par la politique monétaire du gouvernement Thatcher - qui conduit à une contraction rapide de l'emploi manufacturier et affaiblit les bastions traditionnels du mouvement syndical britannique en même temps que le chômage de masse rend les travailleurs et les travailleuses moins prompts à porter des revendications et à recourir à la grève. De plus, les dynamiques à l'œuvre dans les années 1970 ne s'interrompent pas brutalement à la fin de la décennie, et l'on retrouve dans un conflit comme la grève des mineurs de 1984-1985 les caractéristiques que nous avons pu identifier dans la conflictualité sociale des années 1970: affrontement direct entre un syndicat et un gouvernement; mobilisation de l'appareil d'État pour briser la grève; rhétorique anti-syndicale relayée abondamment par les médias ; tensions entre une base radicalisée et une direction qui cherche une issue au conflit. Sur le plan de l'histoire du mouvement syndical, les années 1970 semblent donc commencer à la fin des années 1960 pour s'achever au milieu des années 1980 .

\section{Syndicats et gouvernement : tensions au sein du mouvement travailliste}

11 Si l'on revient au cadrage chronologique strict de la décennie, 1970-1979, alors un même schéma semble se répéter en amont de chacune des deux élections qui portent les conservateurs au pouvoir. En 1964 comme en 1974, un gouvernement travailliste dirigé par Harold Wilson arrive au pouvoir sur un programme de modernisation de l'appareil productif et de redistribution en faveur des classes populaires et en mettant en avant sa proximité avec le mouvement syndical. En 1970 comme en 1979, la fin du mandat est marquée par la déception réciproque entre gouvernement et syndicats, après que les promesses de modernisation et de redistribution ont fait long feu et que des politiques visant à contenir les revendications syndicales se heurtent à la résistance des organisations de travailleurs et de travailleuses. Pour retracer le chemin qui a abouti à l'« hiver du mécontentement » de 1978-1979, il convient donc d'examiner l'évolution des relations entre le mouvement syndical et le parti travailliste - les deux ailes du mouvement travailliste - entre la défaite de 1970 et celle de 1979. En effet, l'« hiver du mécontentement" a ceci de particulier qu'au lieu de mettre aux prises le mouvement syndical avec son ennemi traditionnel, le parti conservateur, il consiste en une série de 
confrontations entre des acteurs qui, syndicats comme gouvernement, font partie d'un même mouvement politique.

\section{Le renouveau de l'alliance entre le parti travailliste et les syndicats}

Suite à la défaite électorale des travaillistes en 1970, les relations tumultueuses entre la direction du parti et les syndicats laissent la place à un front commun contre l'offensive anti-syndicale menée par les conservateurs, sous la forme d'un TUC - Labour Party Liaison Committee, qui permet l'élaboration d'un programme conjoint que les travaillistes peuvent mettre en avant lors des élections de 1974, face à des conservateurs qui buttent contre la résistance que leur oppose le mouvement syndical ${ }^{16}$. Le pilier intellectuel de ce programme commun est le "contrat social», formule imaginée en 1972 par James Callaghan pour désigner les mesures en faveur des classes populaires, des salariés et de leurs syndicats auxquelles le parti travailliste s'engage pour obtenir en retour un engagement des syndicats à la modération dans les revendications salariales ${ }^{17}$. Une fois au pouvoir, le gouvernement Wilson abroge rapidement l'Industrial Relations Act des conservateurs pour lui substituer une législation bien plus favorable aux syndicats et aux salariés, sous la forme du Trade Union and Labour Relations Act de 1974 et de l'Employment Protection Act de 1975, cependant que les dispositions de l'Equal Pay Act de 1970 entrent enfin en vigueur en 1975 et sont complétées la même année par un Sex Discrimination Act en faveur des salariées. La même année cependant, l'inflation atteint $24,05 \%$, provoquant l'inquiétude des détenteurs de livre sterling. Si l'on peut voir dans cette inflation record avant tout l'effet sur l'économie britannique de la flambée mondiale des prix du pétrole et des matières premières ainsi que des mesures en faveur de la croissance de l'économie adoptées par les conservateurs dans leur dernière année au pouvoir, ce ne sont pas des facteurs sur lesquels le gouvernement britannique peut agir s'il souhaite maintenir l'orientation internationale de l'économie britannique et préserver l'attractivité de la livre sterling. Les augmentations salariales, qu'il est alors devenu relativement consensuel de considérer comme un facteur d'inflation, sont en revanche un domaine dans lequel le gouvernement peut agir, précisément en raison du lien qui l'unit au mouvement syndical et de l'esprit qui préside au contrat social. La modération salariale est aussi l'outil par lequel le gouvernement travailliste, comme son prédécesseur conservateur, espère que les entreprises pourront libérer une plus grande marge de profit pour attirer des capitaux et réaliser des investissements productifs permettant de relancer l'économie britannique en perte de vitesse par rapport à ses concurrentes. De l'été 1975 à l'hiver 1978-1979, quatre phases successives de contrôle des revenus se succèdent, qui transforment progressivement l'ambitieux «contrat social» en une politique des revenus ${ }^{18}$.

\section{Entre gouvernement et salariés, le conflit de loyauté des directions syndicales}

13 Un des aspects les plus remarquables de la période 1975-1978 est la faible contestation dont la politique des revenus, qui conduit pourtant à une redistribution des profits du travail vers le capital, fait l'objet jusqu'aux grèves de l'« hiver du mécontentement ». Les syndicats des services publics sont certes, pour la plupart, résolument hostiles à la politique des revenus parce qu'elle s'applique de manière plus stricte dans un secteur 
public dont le budget est contrôlé directement par le gouvernement, mais ils disposent à cette époque d'une position marginale au sein du TUC. Ce sont les directions des grands syndicats manufacturiers - la TGWU (Transport and General Workers Union), l'AUEW ( Amalgamated Union of Engineering Workers) et la GMWU (General and Municipal Workers Union ) - qui déterminent l'orientation du TUC, or ceux-ci ont contribué à l'élaboration de la première phase de la politique de revenus et à son acceptation au congrès du TUC. Leur priorité est alors en effet d'obtenir du gouvernement qu'il mette en œuvre la politique industrielle promise aux syndicats dans le contrat social, c'est-à-dire qu'il favorise l'investissement productif pour enrayer le déclin du secteur manufacturier et la contraction de l'emploi dans ce secteur. À mesure que la politique des revenus conduit à une baisse réelle des revenus des salariés, la contestation des bases militantes contraint toutefois les directions syndicales à modérer leur soutien au gouvernement. En 1978, le gouvernement perd ses appuis syndicats les plus puissants, lors que Jack Jones et Hugh Scanlon, qui dirigeaient respectivement la TGWU et l'AUEW, prennent leur retraite. Au congrès annuel du TUC début septembre 1978, toute idée de poursuite de la politique des revenus est ainsi dénoncée, mais aucune action n'est ouvertement évoquée contre la limitation drastique des augmentations salariales pour 1978-1979, fixée par le gouvernement à $5 \%$ : les syndicats anticipent une élection à l'automne, que les travaillistes doivent gagner pour éviter un retour au pouvoir des conservateurs menés par la très radicale Margaret Thatcher ${ }^{19}$.

Surprenant les calculs de ses amis comme de ses adversaires, le Premier ministre James Callaghan n'organise pas d'élection à l'automne 1978, tablant sur une amélioration de la conjoncture économique du pays d'ici le printemps 1979. Le congrès annuel du parti travailliste, du début du mois d'octobre, se déroule dans une ambiance bien plus acrimonieuse, avec en toile de fond le conflit social dans les usines Ford: une motion s'opposant à la politique des revenus par le gouvernement travailliste, déposée par une section locale, est adoptée à une majorité des deux tiers. On ne peut toutefois qu'être frappé par le caractère relativement apaisé des relations entre les directions syndicales et le gouvernement travailliste au cours de l'hiver 1978-1979. Le gouvernement fait d'ailleurs pression sur la direction de la TGWU, aussi bien dans le conflit dans les usines Ford que dans celui dans les transports routiers, pour que le syndicat apporte le plus rapidement possible son soutien officiel aux grévistes et prenne en retour le contrôle du mouvement. Le 18 janvier 1979, la TGWU publie ainsi un code de conduite à destination des piquets de grève, soulignant la nécessité de laisser passer les chargements de denrées alimentaires et de médicaments. Des responsables du syndicat se réunissent quotidiennement avec les fonctionnaires de la Civil Contingencies Unit, et s'emploient à résoudre les situations problématiques que ceux-ci portent à leur connaissance. De son côté, la direction du TUC s'emploie à jouer un rôle de médiateur entre syndicats et gouvernement tout au long du conflit.

$\mathrm{Du}$ point de vue des syndicats, les conflits de l'hiver 1978-1979 sont donc des protestations organisées en vue d'obtenir des concessions du gouvernement, et non les manifestations de désordre social qu'y voient la direction du parti conservateur et ses relais dans la presse. D'un point de vue gouvernemental, l'impact social et économique des conflits a pareillement été relativement maitrisé : la population n'a eu à subir aucune pénurie totale de nourriture en dépit du blocage des infrastructures par les routiers, et aucune victime directe des conflits n'a été à déplorer en dépit de l'ampleur du mouvement en plein cœur d'un hiver marqué par des températures glaciales. La 
limitation de l'impact politique des conflits s'avère un exercice plus compliqué pour le gouvernement travailliste et les syndicats.

Le 14 février 1979 la direction du TUC et le gouvernement publient une déclaration commune publiée, intitulée The Economy, the Government and Trade Union Responsibilities. Immédiatement affublée du nom de "concordat », cette déclaration fait l'apologie de la concertation et rappelle la primauté des intérêts de la nation britannique dans son ensemble ${ }^{20}$. Ce coup médiatique n'est pas entièrement convaincant, alors que le conflit se durcit dans les services publics. L'annonce faite en février 1979 par la direction de NUPE ( National Union of Public Employees), le plus radical des syndicats des services publics, qu'il reconsidère son affiliation au parti travailliste, révèle ainsi le fossé profond qui s'est creusé entre ce syndicat et le gouvernement. Il n'en reste pas moins que NUPE participe également, tout au long du conflit dans les collectivités locales et le NHS, à des négociations entre les directions syndicales et le gouvernement. Fin janvier, les directions des syndicats des services publics rencontrent le ministre de la santé David Ennals pour mettre au point un "code de conduite» rappelant la nécessité d'assurer en toutes circonstances les services d'urgence ${ }^{21}$.

Ce conflit de loyauté entre les revendications des adhérents et le soutien au gouvernement, dont ce dernier n'hésite pas à jouer devant les dirigeants syndicaux, est cependant dangereux pour les travaillistes au pouvoir. Si les dirigeants syndicaux tendent à privilégier au final la résolution rapide des conflits et la modération de leur impact sur l'image du gouvernement travailliste, il n'est pas aussi sûr que les adhérents des organisations fassent le même choix. Le 26 septembre 1978, les responsables des intersyndicales des deux usines Ford de Dagenham adressent un courrier au Premier ministre, dans lequel ils disent vouloir lui faire part du «sentiment profond de trahison » ressenti par les travailleurs de Ford. Ils constatent une hostilité croissante de leurs collègues à l'égard du gouvernement travailliste et à l'idée d'un soutien syndical au parti lors d'élections futures. Selon eux, le gouvernement commet un «suicide politique » en s'acharnant à maintenir un contrôle des revenus qui pénalise les travailleurs ${ }^{22}$.

\section{La crise politique et intellectuelle du parti travailliste}

Les syndicalistes de Ford font clairement porter au gouvernement travailliste la responsabilité des conflits sociaux liés à la politique de contrôle des revenus, et in fine la désaffection de l'électorat à son égard. Ce débat sur les responsabilités respectives des syndicats et du gouvernement dans l'épisode de l'« hiver du mécontentement » et l'arrivée au pouvoir des conservateurs en mai 1979 reflète plus largement la ligne de fracture qui s'est creusée au sein du parti travailliste, entre une aile gauche qui accuse le gouvernement de trahir son électorat populaire, et une aile qui considère que ce sont les revendications des syndicats qui mettent en danger aussi bien les performances de l'économie britannique que les chances de succès électoral du parti. Les fortes tensions politiques et programmatiques qui traversent le parti tout au long de la décennie ont donné leur tonalité aussi bien aux conflits de l'«hiver du mécontentement» qu'aux débats ultérieurs sur la direction que le parti devait prendre. 


\section{Gauche contre droite, et autres divisions}

19 Bien que les luttes de programme et de pouvoir au sein du parti travailliste opposent un nombre considérable de factions, on peut les rassembler de manière efficace en une division principale entre gauche et droite qui remonte à la période de gouvernement travailliste de l'immédiat après-guerre, 1945-1951. La gauche, derrière la figure charismatique d'Aneurin Bevan, est favorable à une poursuite de la transition du pays vers le socialisme, qu'auraient simplement esquissée les nationalisations et le développement de l'État social après 1945. La droite quant à elle, autour du leader historique Clement Attlee puis de Hugh Gaitskell qui lui succède en 1955, estime que les réformes de la période 1945-1951 ont créé les conditions de la prospérité et du progrès social sous la forme d'une économie mixte et des services sociaux bénéficiant à l'ensemble de la population. Cet antagonisme durable, temporairement affaibli pendant la seconde moitié des années 1960 lorsque le parti est dominé par la figure centriste et modernisatrice de Wilson, revient en force au tournant des années 1970 lorsque les performances économiques et la situation de l'emploi commencent à se dégrader ${ }^{23}$.

Pour l'aile gauche travailliste, l'expérience du gouvernement Wilson de 1964-1970, dont les aspirations à la modernisation de l'économie britannique ont été sabordées par la priorité donnée au maintien d'une livre forte, démontre la nécessité pour le Royaume-Uni de récupérer sa souveraineté économique. Contre les crises monétaires à répétition auxquelles l'orientation internationale de l'économie britannique l'expose, et contre le rôle croissant que les multinationales sont en mesure de jouer dans l'investissement et l'emploi, la gauche formule une politique économique alternative (AES, Alternative Economic Strategy) qui repose sur l'adoption de mesures protectionnistes et la nationalisation des principales entreprises, ainsi que sur une politique industrielle de soutien à la production et à l'emploi À l'inverse, la droite refuse catégoriquement de remettre en cause l'orientation internationale de l'économie qui bénéficie au secteur financier comme aux industries d'exportation, ainsi que de promouvoir des politiques interventionnistes qui menaceraient le principe de l'économie mixte et conduiraient à un affrontement avec les intérêts financiers et industriels. Bien au contraire, la principale crainte des investisseurs, à savoir l'inflation, doit être la priorité des gouvernements, lesquels disposent pour la gérer des instruments traditionnels « keynésiens » que sont la politique fiscale permettant la relance ou le ralentissement de l'économie, ainsi que la régulation des prix et des augmentations salariales ${ }^{24}$.

Ce débat politique recoupe les tensions multiples au sein d'un parti travailliste qui a toujours été fondamentalement un mouvement réunissant des composantes hétérogènes : le parti parlementaire, les syndicats et les sections locales se retrouvent tous les ans lors d'un congrès annuel qui constitue l'instance décisionnelle suprême du parti, mais chacune de ces composantes continue de jouir d'une autonomie relative. Alors que la droite, qui domine la direction du parti depuis l'entre-deux-guerres, a pu compter tout au long de l'après-guerre sur le soutien des directions des principaux syndicats britanniques, celles-ci basculent à gauche au tournant des années 1970 avec l'arrivée à la tête de l'AUEW et de la TGWU de Hugh Scanlon et de Jack Jones. Ceux-ci, bien qu'animés tout au long de la décennie par l'impératif de porter ou de maintenir le parti travailliste au pouvoir, promeuvent des politiques économiques interventionnistes en faveur du secteur industriel. Les sections locales du parti (Constituency Labour Parties), qui avaient déjà 
constitué une base de soutien pour la gauche à l'époque de Bevan, voient arriver dans leurs rangs au cours des années 1970 une génération de jeunes militant-e-s davantage acquis aux thèmes développés par l'aile gauche du parti.

\section{De 1974 à 1979, une série de crises autour de l'orientation économique du gouvernement}

Ces tensions multiples entre orientations programmatiques et composantes, dont l'impact demeure modéré lorsque le parti est uni dans son opposition au gouvernement conservateur d'Edward Heath, connaissent une accélération après le retour au pouvoir des travaillistes en 1974. Un premier épisode en est le référendum de 1975 sur l'appartenance du Royaume-Uni à la Communauté économique européenne (CEE), négociée par les conservateurs et entrée en vigueur au $1^{\mathrm{er}}$ janvier 1973 : symbolique de l'approfondissement de l'orientation internationale de l'économie et de la mise en concurrence des industries britanniques avec leurs homologues européennes, cette question divise le parti selon un axe droite-gauche. La victoire écrasante des partisans de la CEE permet à Harold Wilson de marginaliser Tony Benn, qui s'était imposé comme le porte-étendard de la gauche et de son programme d'AES, et d'enterrer les projets de politique industrielle ambitieuse que celui-ci portait au sein du gouvernement. L'année suivante est marquée par la «crise du FMI» (Fonds monétaire international), lorsque ce dernier impose au gouvernement travailliste des coupes drastiques dans les dépenses publiques en échange d'un prêt permettant au gouvernement de rassurer les détenteurs de livres sterling sur la santé économique du pays. Les réductions budgétaires demandées par le FMI ne feraient jamais qu'amplifier la politique de réduction des dépenses publiques déjà engagée par le Chancelier de l'Échiquier Denis Healey avec le soutien du nouveau Premier ministre James Callaghan, mais la soumission du gouvernement travailliste aux volontés d'une organisation internationale enrage la gauche du parti ${ }^{25}$.

La période entre 1976 et 1978, qui mène aux conflits sociaux de l'hiver 1978-1979, est donc marquée par de fortes tensions au sein du parti travailliste : le congrès annuel du parti travailliste vote chaque année des résolutions en faveur d'une politique économique de type AES, en rupture avec l'orientation de la politique du gouvernement. Les syndicats des services publics dénoncent, depuis les marges du mouvement syndical, l'impact de la réduction des dépenses publiques sur l'emploi, les conditions de travail et les usagers, cependant que les syndicats du secteur manufacturier qui dominent le TUC tentent vainement d'obtenir du gouvernement des mesures de protection en faveur de l'industrie britannique ${ }^{26}$. Les désaccords entre syndicats et gouvernement dépassent donc largement la seule question du contrôle des revenus, même si celle-ci demeure le principal point d'achoppement. L'amélioration de la conjoncture économique en 1978, et le relâchement de la pression budgétaire sur les services publics au cours de la même année, ne permet pas de compenser l'animosité qui s'est développée dans la base militante des syndicats. Cet ensemble de tensions jouent pleinement dans l'affrontement de l'«hiver du mécontentement» entre un gouvernement arc-bouté sur sa stratégie de lutte contre l'inflation et des syndicats qui chercher à en infléchir la trajectoire. 


\section{Conclusion}

24 retrait $\mathrm{du}$ soutien des partis nationalistes écossais et gallois après l'échec des référendums sur la dévolution. Cependant, la position minoritaire dans laquelle les travaillistes se trouvent à la Chambre des communes depuis l'automne 1976 est bien le résultat d'une série de défaites électorales lors d'élections partielles qui ont entamé, puis annulé, la majorité dont le parti bénéficiait à la Chambre des communes depuis l'élection d'octobre 1974. L'« hiver du mécontentement ", comme moment de désunion maximale entre les ailes parlementaire et syndicale du mouvement travailliste, semble donc bien n'être qu'une étape, aussi marquante soit-elle, dans la fracture du lien entre le gouvernement travailliste et son électorat.

Au final, si l'«hiver du mécontentement» est bien un moment de crise pour le mouvement ouvrier britannique comme pour le Royaume-Uni, c'est au sens d'un moment où les projets politiques contradictoires portés par les différents acteurs se heurtent frontalement: l'hypothèse privilégiée par la droite du parti travailliste, à savoir la poursuite d'une gestion des difficultés économiques et monétaires du Royaume-Uni par la politique fiscale et la régulation des prix et des salaires, fait face aux demandes de réorientation économique formulées par l'aile gauche du parti travailliste, et à la rupture néo-libérale désormais avancée ouvertement par le parti conservateur. Ce sont au final les chocs intérieurs et extérieurs de la période 1979-1983, tout particulièrement la formation du SDP (Social Democratic Party) suite à la scission d'une partie l'aile droite du parti travailliste, ainsi que le regain patriotique autour de la Guerre des Malouines, qui tranchent entre ces différentes trajectoires possibles en permettant au parti conservateur d'accroître sa position de force et en résolvant la crise interne du travaillisme en faveur de l'aile droite du parti.

\section{BIBLIOGRAPHIE}

Coates Ken et Topham Tony, Trade Unions in Britain, Nottingham, Spokesman, 1980.

Cronin James, Industrial Conflict in Modern Britain, Londres, Croom Helm, 1979.

Gamble Andrew, Britain in Decline. Economic Policy, Political Strategy and the British State, New York, St Martin's

Hay Colin, « Narrating Crisis: The Discursive Construction of the Winter of Discontent ", Sociology, vol. 30, No. 2 (1996), p. 253-277.

Hickson Kevin, The IMF Crisis of 1976 and British Politics, Londres, Tauris Academic Studies, 2005.

Holmes Martin, The Labour Government, 1974-79. Political Aims and Economic Reality, Basingstoke, Macmillan, 1985.

Revue Française de Civilisation Britannique, XXII- Hors série | 2017 
Lenormand Marc, «Une histoire critique de l'“hiver du mécontentement” de 1978-1979 : Le mouvement syndical britannique face à la crise du travaillisme, l'extension de la conflictualité sociale et la montée de la nouvelle droite thatchérienne " [en ligne], Université Lumière Lyon 2, 2012, mis en ligne 2012, consulté le 29 septembre 2016. URL : http://theses.univ-lyon2.fr/ documents/getpart.php?id=3051\&action=pdf.

Ludlam Steve, «Labourism and the disintegration of the postwar consensus : disunited trade union economic policy responses to public expenditure cuts, 1974-1979», PhD thesis, University of Sheffield, 1990.

Martin López Tara, The Winter of Discontent : Myth, Memory, and History, Liverpool, Liverpool University Press, 2014.

Minkin Lewis et Seyd Patrick, "The British Labour Party ", in Paterson William et Thomas Alastair (dir.), Social Democratic Parties in Europe, Londres, Croom Helm, 1977, p. 103-145.

Reid Alastair et Pelling Henry, A Short History of the Labour Party, Basingstoke, Palgrave Macmillan, 2005.

Shepherd John, Crisis? What crisis? The Callaghan Government and the British 'Winter of Discontent', Manchester, Manchester University Press, 2013.

Smith Paul, « The 'Winter of Discontent' : The Hire and Reward Road Haulage Dispute, 1979 », Historical Studies in Industrial Relations, No. 7 (1999), p. 27-54.

Taylor Andrew, «The Conservative Party and the Trade Unions », Mcllroy John et al. (dir.), The High Tide of British Trade Unionism: trade unions and industrial politics, 1964-1979, Monmouth, Merlin, 2007, p. 151-186.

Taylor Robert, The Trade Union Question in British Politics: Government and Unions since 1945, Oxford, Blackwell, 1993.

Thompson Noël, Political Economy and the Labour Party. The economics of democratic socialism, 1884-2005, Oxford, Routledge, 2006.

Thorpe Andrew, « The Labour Party and the Trade Unions », in Mcllroy John et al. (dir.), The High Tide of British Trade Unionism: trade unions and industrial politics, 1964-1979, Monmouth, Merlin, 2007, p. 133-150.

Tomlinson Jim, « Economic Policy », in Seldon Anthony et Hickson Kevin (dir.), New Labour, Old Labour: The Wilson and Callaghan Governments, 1974-1979, Londres, Routledge, 2004, p. 55-69.

Trades Union Congress, Report of $111^{\text {th }}$ Annual Trades Union Congress, Londres, TUC, 1979.

Williams Stephen et Fryer Robert, Leadership and Democracy: The History of the National Union of Public Employees, Vol. 2 « 1928-1993 », Londres, Lawrence \& Wishart, 2011, p. 168-192.

\section{NOTES}

1. On parle de politique des revenus (incomes policy) pour désigner la limitation par le gouvernement des augmentations salariales que les syndicats sont susceptibles de négocier avec les employeurs.

2. Ces conflits ont été décrits de manière précise dans deux ouvrages publiés récemment : MARTIN LÓPEZ Tara, The Winter of Discontent : Myth, Memory, and History, Liverpool, Liverpool University Press, 2014 ; SHEPHERD John, Crisis? What crisis? The Callaghan Government and the British 'Winter of Discontent', Manchester, Manchester University Press, 2013. 
3. On parle de "recentrage" pour qualifier le repositionnement de partis ou de syndicats au centre de l'échiquier politique. Ce processus, qui passe par l'abandon des parties les plus radicales de leur programme, a été tout particulièrement marqué au sein du parti travailliste sous les directions de Tony Blair (1994-2007) et de Gordon Brown (2007-2010), même s'il a commencé lorsque Neil Kinnock (1983-1992) a lancé la « modernisation » du parti.

4. Voir le chapitre «Crosscurrents : Myth, Memory and Counter-Memory » dans MARTIN LOPEZ Tara, op.cit., 2014.

5. HAY Colin, "Narrating Crisis: The Discursive Construction of the Winter of Discontent", Sociology, vol. 30, No. 2 (1996), p. 253-277.

6. Hay emprunte la notion d'« hégémonie " au penseur marxiste italien Antonio Gramsci. Elle désigne le moment, antérieur à la conquête du pouvoir politique, où l'idéologie d'une classe ou d'une fraction d'une classe jusqu'alors en position subordonnée s'impose comme nouvelle idéologie dominante, facilitant et accélérant son arrivée au pouvoir.

7. Cet article croise une synthèse des ouvrages de référence sur la période et le produit de recherches en archive réalisées pour notre thèse de doctorat. Pour une liste des archives utilisées, voir les annexes de LENORMAND Marc, " Une histoire critique de l'"hiver du mécontentement" de 1978-1979 : Le mouvement syndical britannique face à la crise du travaillisme, l'extension de la conflictualité sociale et la montée de la nouvelle droite thatchérienne » [en ligne], Université Lumière Lyon 2, 2012, mis en ligne 2012, consulté le 29 septembre 2016. URL : http://theses.univ-lyon2.fr/documents/getpart.php?id=3051\&action=pdf. 8. THORPE Andrew, "The Labour Party and the Trade Unions", in MCILROY John et al. (dir.), The High Tide of British Trade Unionism: trade unions and industrial politics, 1964-1979, Monmouth, Merlin, 2007, p. 136.

9. TAYLOR Andrew, «The Conservative Party and the Trade Unions ", MCILROY John et al. (dir.), The High Tide of British Trade Unionism: trade unions and industrial politics, 1964-1979, Monmouth, Merlin, 2007, p. 158-159.

10. Holmes Martin, The Labour Government, 1974-79. Political Aims and Economic Reality, Basingstoke, Macmillan, 1985, p. 119-20; TAYLOR Robert, The Trade Union Question in British Politics: Government and Unions since 1945, Oxford, Blackwell, 1993, p. 249.

11. Cette grève, qui part de la protestation d'un groupe de femmes originaires d'Asie du sud et d'Afrique orientale, contre leur renvoi et pour l'amélioration des conditions de travail dans l'usine, prend une dimension nationale lorsqu'elles essaient d'obtenir la reconnaissance par leur employeur de droits syndicaux à l'usine. Le soutien qu'elles reçoivent du mouvement syndical dans son ensemble, sous la forme de piquets de grève massifs qui conduisent à des affrontements avec la police, ne parvient pas cependant à faire plier l'employeur, soutenu quant à lui par les organisations anti-syndicales qui gravitent autour du parti conservateur.

12. coATes Ken et торнам Tony, Trade Unions in Britain, Nottingham, Spokesman, 1980, p. 217; CRONIN James, Industrial Conflict in Modern Britain, Londres, Croom Helm, 1979, p. 185.

13. WILLIAms Stephen et Fryer Robert, Leadership and Democracy: The History of the National Union of Public Employees, Vol. 2 «1928-1993 », Londres, Lawrence \& Wishart, 2011, p . 168-192.

14. SMITH Paul, « The 'Winter of Discontent' : The Hire and Reward Road Haulage Dispute, 1979 », Historical Studies in Industrial Relations, No. 7 (1999), p. 27-54.

15. WILLIAMS Stephen et FRYER Robert, op. cit., 2011, p. 310-377.

16. MINKIN Lewis et SEYD Patrick, "The British Labour Party ", in PATERSON William et THOMAS Alastair (dir.), Social Democratic Parties in Europe, Londres, Croom Helm, 1977, p. 103-145.

17. TAYLOR Robert, op. cit., 1993, p. 226

18. TOMLINSON Jim, «Economic Policy ", in SELDON Anthony et HICKSON Kevin (dir.), New Labour, Old Labour: The Wilson and Callaghan Governments, 1974-1979, Londres, Routledge, 2004, p. 55-69. 
19. TAYLOR Robert, op. cit., 1993, p. 251-252.

20. TRADES UNION CONGRESS, Report of $111^{\text {th }}$ Annual Trades Union Congress, Londres, TUC, 1979, p. 274.

21. WILLIAMS Stephen et FRYER Robert, op. cit., 2011, p. 329-330.

22. « Letter from the convenors of the Ford Dagenham River Plant and the Ford Dagenham Body Plant to the Prime Minister, September 26 ${ }^{\text {th }}, 1978 »$, TGWU Archive, Modern Records Centre, University of Warwick, MSS.126/TG/466/A1/1/4/6.

23. Sur ces questions, voir REID Alastair et PELLING Henry, A Short History of the Labour Party, Basingstoke, Palgrave Macmillan, 2005, tout particulièrement p. 132-152.

24. GAMBLE Andrew, Britain in Decline. Economic Policy, Political Strategy and the British State, New York, St Martin's, 1994. p. 157-185; тHоMPSON Noël, Political Economy and the Labour Party. The economics of democratic socialism, 1884-2005, Oxford, Routledge, 2006, p. 189-234.

25. HICKSON Kevin, The IMF Crisis of 1976 and British Politics, Londres, Tauris Academic Studies, 2005. 26. LUDLAM Steve, «Labourism and the disintegration of the postwar consensus : disunited trade union economic policy responses to public expenditure cuts, 1974-1979 », PhD thesis, University of Sheffield, 1990.

\section{RÉSUMÉS}

Le mythe politique que l'« hiver du mécontentement » de 1978-1979 en est venu à constituer dans la mémoire collective et le débat d'idées au Royaume-Uni a désormais fait l'objet d'un travail de démystification, controversé certes, mais remettant fermement en cause la perception de cet épisode comme un moment de désordre social appelant la remise en ordre thatchérienne. Cet article s'emploie à mettre en évidence une série de processus qui, sur l'ensemble de la décennie, permettent de rendre compte de la série d'affrontements fratricides, à l'hiver 1978-1979, entre des syndicats et un gouvernement que rassemble pourtant l'appartenance à un même parti politique: la transformation du mouvement syndical à partir de la fin des années 1960 ; l'évolution de la relation entre les ailes syndicales et parlementaire du mouvement travailliste entre 1970 et 1979 ; enfin, les tensions politiques et programmatiques très fortes qui traversent le parti travailliste pendant cette même période. L' « hiver du mécontentement » apparaît au final comme une étape dans la crise interne du mouvement travailliste, qui ne connaît une résolution au profit de la droite du parti que dans les années 1980.

The 1978-1979 "winter of Discontent » has now been thoroughly analysed as a political myth through which the industrial disputes of the winter 1978-1979 were defined as the sure sign of a larger crisis of state authority and of the social-democratic management of the economy, thus helping usher in the Conservatives' neoliberal agenda. This paper highlights a series of dynamics running through the whole decade 1970-1979, which led to the industrial disputes of 1978-1979 pitting trade unions against a Labour government, thereby revealing the fracturing of the alliance between the industrial and political wings of the UK Labour movement. These dynamics are the internal transformation of the trade union movement, the evolving relation between trade unions and government in opposition then in power, and finally the increasing divide between left and right within the party as the leadership of the party rejected the calls for an Alternative Economic Strategy and introduced strict limits on public spending and wage increases in the second half of the decade. The "winter of Discontent » can therefore best be 
conceived as a stage, albeit climactic, in the process of resolving the internal crisis of Labour, which took the form of « modernisation » and a shift to the centre from the mid-1980s.

INDEX

Keywords : trade unionism, strikes, « winter of discontent ", James Callaghan, 1970s

\section{AUTEUR}

MARC LENORMAND

EMMA, EA741, Université Paul-Valéry Montpellier 3, 